\title{
MEG studies of motor cortex gamma oscillations: evidence for a gamma "fingerprint" in the brain?
}

\author{
Douglas Cheyne $^{1 *}$ and Paul Ferrari ${ }^{2}$ \\ 1 Program in Neurosciences and Mental Health, Hospital for Sick Children Research Institute, Toronto, ON, Canada \\ ${ }^{2}$ Radiology Department, Meadowlands Hospital Medical Center, Secaucus, NJ, USA
}

\author{
Edited by: \\ Krish Singh, Cardiff University, UK \\ Reviewed by: \\ Alfons Schnitzler, \\ Heinrich-Heine-University, Germany \\ Jyrki Mäkelä, Hospital district of \\ Helsinki and Uusimaa, Finland \\ *Correspondence: \\ Douglas Cheyne, Program in \\ Neurosciences and Mental Health, \\ Hospital for Sick Children Research \\ Institute, 555 University Avenue, \\ Toronto, ON M5G 1X8, Canada \\ e-mail: douglas.cheyne@utoronto.ca
}

The human motor cortex exhibits transient bursts of high frequency gamma oscillations in the $60-90 \mathrm{~Hz}$ range during movement. It has been proposed that gamma oscillations generally reflect local intracortical activity. However, movement-evoked gamma is observed simultaneously in both cortical and subcortical (basal ganglia) structures and thus appears to reflect long-range cortical-subcortical interactions. Recent evidence suggests that gamma oscillations do not simply reflect sensory reafference, but have a facilitative role in movement initiation. Here we summarize contributions of MEG to our understanding of movement-evoked gamma oscillations, including evidence that transient gamma bursts during the performance of specific movements constitutes a stereotyped spectral and temporal pattern within individuals-a gamma "fingerprint"-that is highly stable over time. Although their functional significance remains to be fully understood, movement-evoked gamma oscillations may represent frequency specific tuning within cortical-subcortical networks that can be monitored non-invasively using MEG during a variety of motor tasks, and may provide important information regarding cortical dynamics of ongoing motor control.

Keywords: MEG, gamma oscillations, motor cortex, frequency tuning, basal ganglia
Cortical oscillations in the frequency range of $30-90 \mathrm{~Hz}-$ so called gamma band oscillations-can be recorded from a wide range of brain regions during rest or the performance of cognitive-motor tasks, and are assumed to reflect wide-scale neuronal processes associated with cognition and perception (TallonBaudry and Bertrand, 1999; Fries, 2009). Such oscillations are variably time-locked to specific sensory or motor events and such "induced" gamma oscillations have been proposed to reflect global brain mechanisms underlying the attentional control of sensory input (Womelsdorf and Fries, 2007). Oscillations in the lower gamma range can also be elicited or "evoked" by sensory stimulation with modality specific effects, and have been implicated in the binding of low level input within sensory systems that underlies feature processing, particularly in the visual system (Engel and Singer, 2001). Magnetoencephalography (MEG) studies have shown that gamma band oscillations can also be recorded macroscopically from various sensory and motor areas. For example, gamma activity in the $40 \mathrm{~Hz}$ range can be observed in auditory areas during transient or steady-state acoustic stimulation (Pantev et al., 1991; Ross et al., 2005) and speech (Palva et al., 2002). MEG studies have shown sustained gamma oscillations in the $30-60 \mathrm{~Hz}$ range in primary visual cortex during presentation of contrast gratings, which are modulated by a variety of stimulus parameters, such as eccentricity, spatial frequency and orientation (Adjamian et al., 2008; Swettenham et al., 2009; Muthukumaraswamy and Singh, 2013). Thus, gamma oscillations in sensory areas appear to play a specific role in the encoding of stimulus features.
Gamma band activity has also been observed in motor and premotor areas from intracranial depth electrode recordings (Szurhaj et al., 2006) and surface electrocorticogram (ECoG) recordings (Pfurtscheller et al., 2003; Brovelli et al., 2005; Miller et al., 2007). Crone and colleagues (Crone et al., 1998) reported increased gamma activity in the ECoG in awake patients performing sustained muscle contractions. While movement-related gamma band synchronization has been reported in the scalprecorded EEG (Ball et al., 2008; Darvas et al., 2010) such lowamplitude, high-frequency brain activity is highly susceptible to contamination by electromyographic (EMG) activity from scalp, jaw and neck muscles (Whitham et al., 2007) or even microsaccade-generated spike potentials (Yuval-Greenberg et al., 2008). MEG is much less susceptible to such volume conduction artifacts, and thus an ideal method for studying highfrequency cortical activity. However, caution should still be used in interpreting movement-locked gamma oscillations in both EEG and MEG data, particularly if such activity is similar in its frequency profile to simultaneously occurring EMG activity (Muthukumaraswamy, 2013). This can be particularly problematic for speech and orofacial movements, in which case it would be prudent to include EMG surface recordings of scalp muscle activity (Gross et al., 2013). Alternatively, source analysis methods such as minimum-variance beamforming can be used to optimally separate brain and muscle activity, although sources that localize to posterior or inferior frontal locations may still be affected by activity from nearby muscles and should be scrutinized for EMG contamination. 


\section{MOTOR CORTEX GAMMA OSCILLATIONS}

There is abundant evidence that overt movements elicit a specific form of narrow-band, transient oscillatory activity in the range of $70-80 \mathrm{~Hz}$ in the region of the primary motor cortex. This "finelytuned gamma" (Jenkinson et al., 2013) is distinguished from the spectrally broader, induced gamma band oscillations that are typically associated with perceptual or cognitive processes, and is strongly associated with the occurrence of overt movements. Most significantly, narrowly tuned gamma oscillations with similar timing and frequency are observed within various subcortical structures from depth electrodes implanted in patients for the treatment of various motor disorders, including the subthalamic nucleus (Amirnovin et al., 2004; Alegre et al., 2005; Androulidakis et al., 2007; Lalo et al., 2008) globus pallidus (Tsang et al., 2012a) and thalamus (Brucke et al., 2013). Using beamformer source analysis of MEG recordings, we were able to directly observe narrowly tuned gamma band oscillations in motor cortex in healthy subjects during movements of the upper and lower limbs (Cheyne et al., 2008). In comparison to lower frequency beta and mu band oscillations, which are observed bilaterally in MI, motor cortex gamma was highly lateralized to the contralateral primary motor cortex and somatotopically organized. These transient increases in high gamma with movement, sometimes referred to as gamma event-related synchronization (gamma ERS), have been replicated in a number of other MEG studies (Dalal et al., 2008; Tecchio et al., 2008; Muthukumaraswamy, 2010; Hinkley et al., 2011; Gaetz et al., 2013).

Muthukumaraswamy (2010) studied the influence of various kinematic and task parameters on motor cortex gamma, and found that both cued and voluntary movements elicited brief 200-300 ms bursts of gamma activity, even when followed by sustained isometric contractions. Similarly, repetitive movements generated multiple gamma bursts with each movement. Importantly, gamma oscillations did not appear to be simply due to movement-induced somatosensory feedback, since individual gamma bursts were observed for active, but not passive movements at the same rate. Gamma activity preceding the onset of movement has been observed for complex tasks (Gaetz et al., 2013) and self-paced as opposed to visually cued movements in subcortical recordings (Tsang et al., 2012a) and we have also noted variable timing of gamma bursts when inspecting single trial data. Sustained gamma activity has been reported for continuous tracking movements (Kennedy et al., 2011), although we have observed a marked decrement in motor cortex gamma for fast $(>1 \mathrm{~Hz})$ movement rates, indicating that it may be reduced during rhythmic movement (unpublished observations). Bilateral gamma oscillations are elicited by lip movements (Bells et al., 2007) and gamma oscillations have been observed in frontal eye-fields during saccadic eye-movements (Hinkley et al., 2011). Movement-related gamma activity has also been shown to be present in children and adolescents (Gaetz et al., 2010; Wilson et al., 2010; Huo et al., 2011), including children as young as 3.5 years of age (Cheyne, 2013).

Thus, high-frequency, narrowly tuned gamma oscillations are elicited by many types of movement, are strongly linked to movement onset, and appear to reflect activation of neural populations in primary motor cortex. Somatosensory stimulation can also induce high-frequency activity in somatosensory areas (Bauer et al., 2006) raising the possibility that there may be some contributions from SI due to movement-induced sensory feedback. However, movement-related gamma has been consistently localized to regions of the precentral gyrus using beamformer analysis of MEG data, which has been shown to discriminate precentral and postcentral sources of movementrelated activity (Cheyne et al., 2006; Jurkiewicz et al., 2006; Cheyne, 2013). This, combined with the fact movement-related gamma can be observed prior to movement onset, and is not elicited by passive movements alone, suggests that it plays an important role in the generation or monitoring of motor output.

\section{MOTOR GAMMA AND INDIVIDUAL DIFFERENCES}

In our earlier study, we noted a marked similarity in the strength, frequency and duration of gamma oscillations observed in the left and right motor cortex for movements of the same body part in individual subjects (Cheyne et al., 2008). A re-analysis of these data is presented in Figure 1. Using the synthetic aperture magnetometry (SAM) beamformer (Robinson and Vrba, 1999) we generated $4 \mathrm{~mm}$ images of changes in gamma power $(60-90 \mathrm{~Hz})$ relative to pre-movement baseline. Figure 1A shows a gamma increase localized to a focal location in the contralateral primary motor cortex in one subject, and the corresponding Morletwavelet based time-frequency reconstruction of source activity (width $=20$ cycles), demonstrating an extremely narrow-band power increase centered around $80 \mathrm{~Hz}$. We performed bootstrap resampling to estimate the standard errors (SEs) of peak gamma frequency across trials (Muthukumaraswamy and Singh, 2013) and found that peak frequency was highly similar between hemispheres, varying by less than $1 \mathrm{~Hz}$ within individual subjects (Figure 1B). As indicated by the relatively small SEs, gamma frequency was stable across trials, with each subject showing characteristic peak gamma frequency. As reported in the initial study, a high degree of left-right symmetry was also observed for bicep contractions and foot dorsiflexions, with lower gamma frequency for lower compared to upper limb movements. As shown in Figure $\mathbf{2 B}$, the absolute strengths of gamma bursts are small, ranging from 0.5 to $2.0 \mathrm{nAm}^{2}$. Using data simulations we confirmed that the latter would correspond to an oscillating dipole source of about $5 \mathrm{nAm}$ in source strength, generating peak-peak amplitudes at the sensors on the order of about 50 femtoTesla, making such signals difficult to observe in the raw data.

The foregoing observations suggest that motor cortex gamma oscillations are finely tuned in frequency, show similarities for different movement types within individuals, and frequency differences across individuals. This implies that each individual has a characteristic pattern of motor gamma activation-a gamma band "fingerprint"-for specific types of motor output. If so, these patterns should be replicable across recording sessions. To test this hypothesis, we retrospectively analyzed data from two right-handed adults (including one of the authors) in which MEG recordings during left and right self-paced index finger movements (button presses) had been repeated in separate sessions at the same time each day, over a 
A

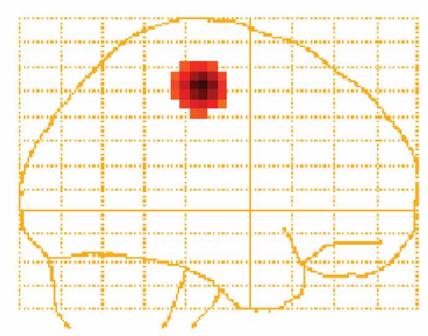

left precentral gyrus (MNI coord $=-26-2050)$

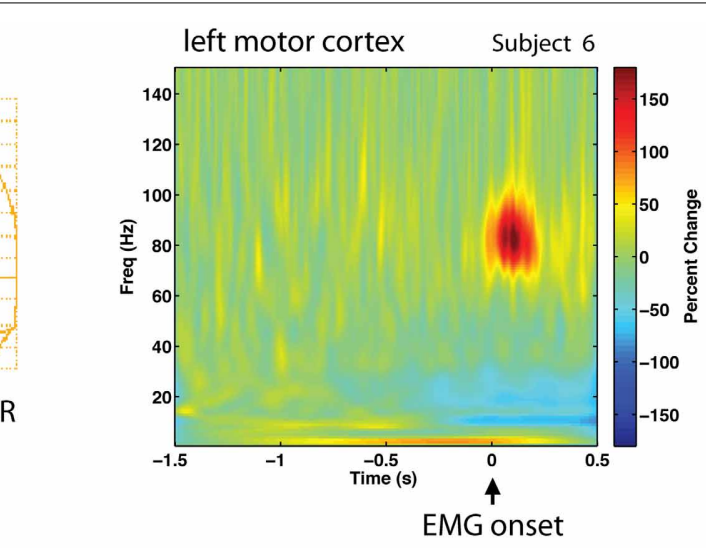

B
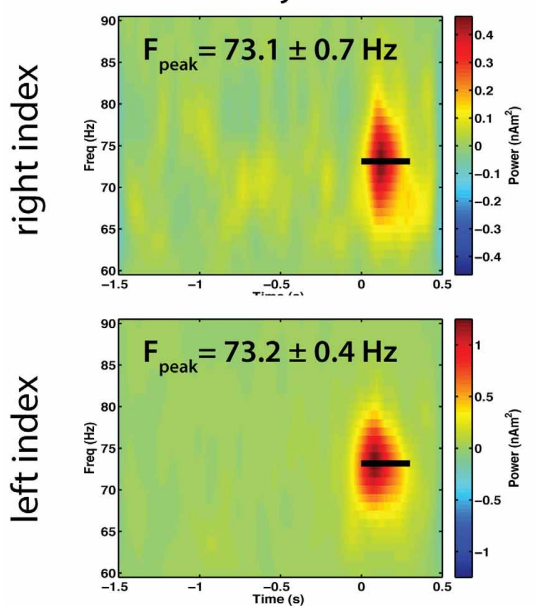

Subject 7

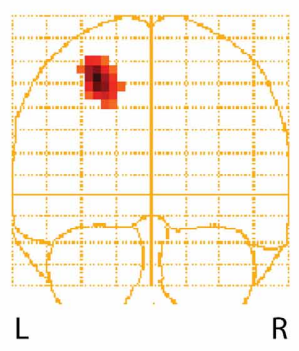

$\mathrm{R}$

Subject 5
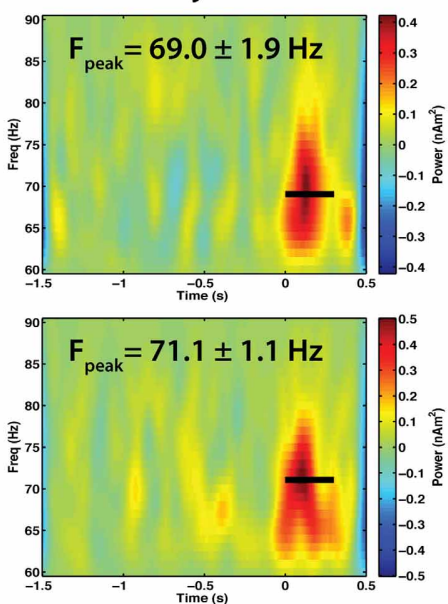
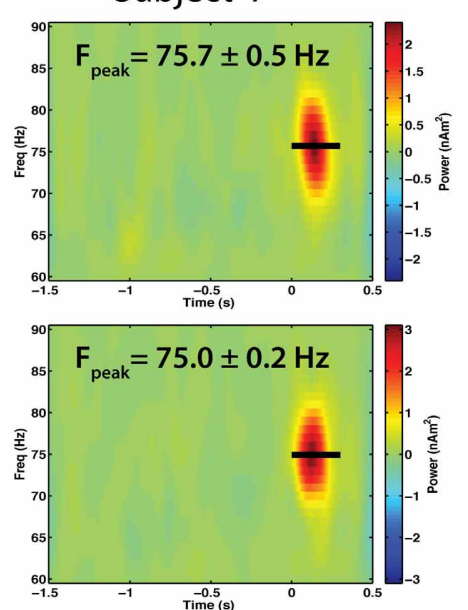

FIGURE 1 | (A) Gamma band responses during right finger abductions in a right-handed subject. Synthetic Aperture Magnetometry (SAM) source localization was used to localize the generator of gamma power in right motor cortex, transformed to $\mathrm{MNI}$ coordinates with $4 \mathrm{~mm}$ resolution (left). A time-frequency plot of the source activity (right) over the range of $1-150 \mathrm{~Hz}$ shows a brief burst of gamma band activity around $80 \mathrm{~Hz}$ immediately following EMG onset ( $t=0 \mathrm{~s}$ ) as a percent change above baseline
$(-1.5$ to $-1 \mathrm{~s})$. (B) Time-frequency analysis of source activity from contralateral motor cortex for left and right index finger movements in three selected right-handed subjects, showing the peak frequency $\left(F_{\text {peak }}\right)$ and standard error in each subject, estimated from 5000 bootstrap resampling (with replacement) of the single trial time-frequency estimates. Beamformer source analysis was carried out using the BrainWave analysis toolbox (http://cheynelab.utoronto.ca). five-day period. In addition, these measurements were available in both subjects recorded $\sim 5$ years earlier, and in one subject 4.5 years later. Figure 2 presents an analysis of motor cortex gamma activity across different sessions for both subjects. Averaged time courses of source activity in contralateral MI, computed using an event-related beamformer (Cheyne et al., 2006), confirmed that the subject's performance was highly consistent across sessions (Figure 2A). Time-frequency plots of source power in MI for left and right movements show gamma bursts beginning at movement onset and lasting $\sim 300 \mathrm{~ms}$ (Figure 2A right). Mean peak frequency and SEs for each hemisphere and session were estimated using bootstrap resampling of single trial time-frequency estimates and compared over all sessions (a total of 8 sessions for Subject 1 and 7 sessions for Subject 2). Figure 2B shows time-frequency plots for the right hand condition in Subject 1, with peak gamma frequency often varying by less than $1 \mathrm{~Hz}$ between sessions.
Figure 2C shows the peak frequency over all sessions for both subjects, demonstrating stability over multiple sessions. Peak gamma frequency was lower for left compared to right index finger movements across sessions for Subject 1, with mean peak frequency $( \pm S E)$ of $71.24 \pm 0.38 \mathrm{~Hz}$ for right finger movements and $68.47 \pm 0.71 \mathrm{~Hz}$ for left finger movements $(p<0.005$, paired $t$ test). Interestingly, the latency of gamma peak frequency was also earlier for left finger movements (79.26 $\pm 9.4 \mathrm{~ms})$ compared to right finger movements $(151.1 \pm 7.5 \mathrm{~ms})$ across all sessions $(p<0.0001$, paired $t$-test). For Subject 2, peak frequency was also consistent for both left and right index finger movements across repeated sessions, with almost identical mean gamma frequency for left $(68.2 \pm 0.4 \mathrm{~Hz})$ and right $(69.8 \pm$ $0.5 \mathrm{~Hz}$ ) finger movements $(p=0.39$, paired $t$-test). Gamma latency was also not significantly different for left and right movements in this subject. Although preliminary, these results suggest that individuals demonstrate highly replicable peak gamma 

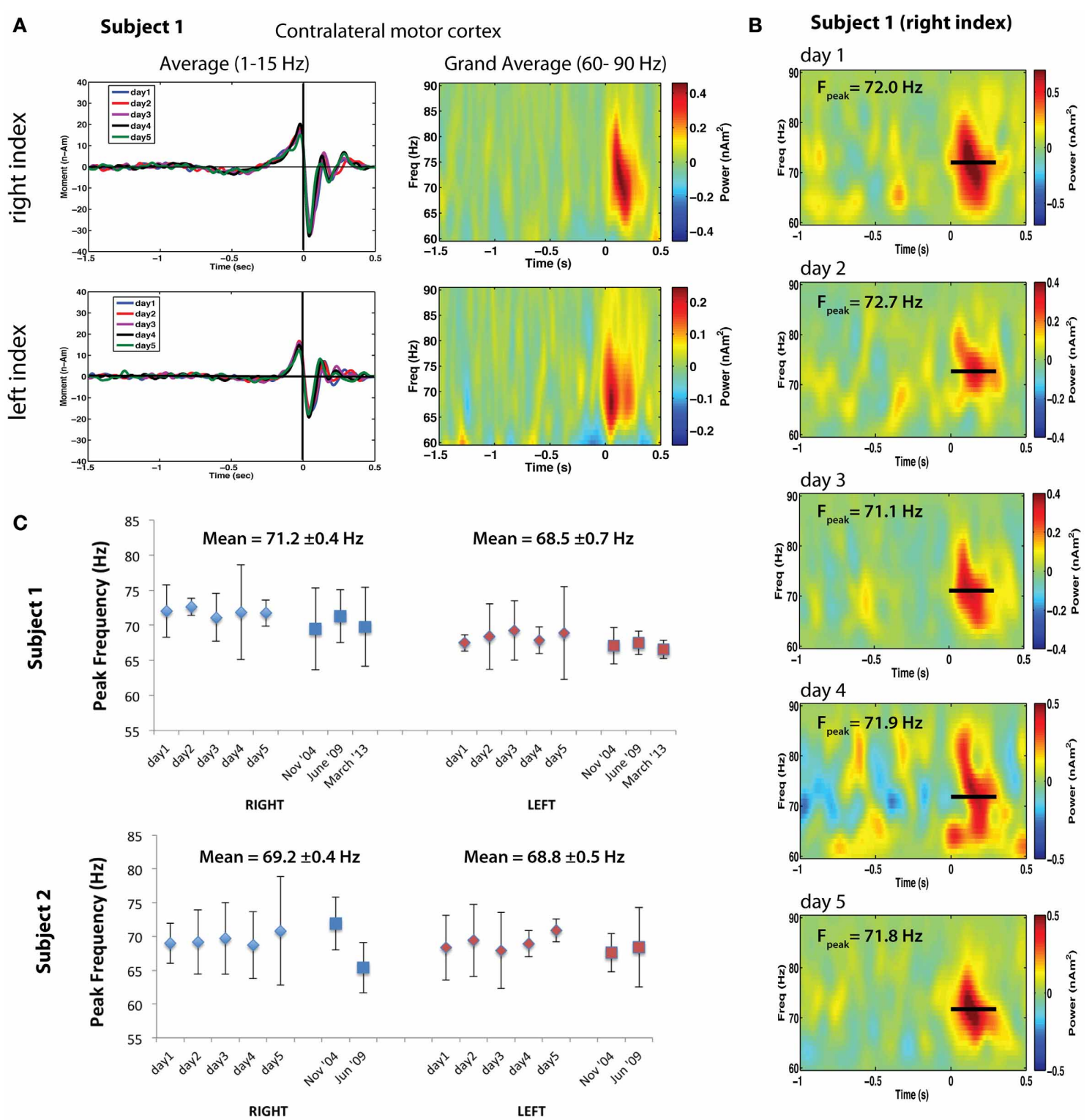

FIGURE 2 | (A) Left: Averaged motor fields for all 5 recording sessions for left and right index finger movements (time-locked to button press). Right: Corresponding time-frequency analysis of the single trial power from 60 to $90 \mathrm{~Hz}$ for the peak locations of gamma activity. (B) Bootstrapped mean time-frequency plots for the 5 repeated sessions for right index finger movements. $F_{\text {peak }}$ indicates the mean peak frequency \pm standard error. (C) Peak motor cortex gamma frequencies for right index finger (RIGHT, blue markers) and left index finger (LEFT, red markers) movements repeated for 5 sessions over a one-week period for two subjects. Sessions taken at different time intervals separated by approximately $4-5$ years are also shown. Each data point indicates the bootstrap resampled mean peak frequency, using 5000 re-samplings of two-thirds of the single trial time-frequency estimates. Error bars indicate the $95 \%$ confidence intervals based on the bootstrap standard error. frequencies in motor cortex for the same movements, and that these are stable over long periods of time, indicative of an inter-individual characteristic frequency of motor cortex gamma oscillations.

\section{THE FUNCTIONAL ROLE OF MOTOR CORTEX GAMMA OSCILLATIONS}

The role of high gamma oscillations in motor control is unknown. It is clear that these oscillations are markedly similar in frequency and latency to those observed in the basal ganglia and other subcortical motor structures, which have reciprocal connections with motor cortex (Shepherd, 2013). Coherence between oscillations in MI and STN are well established for the lower beta band (Brown, 2003; Lalo et al., 2008). However, such long-range synchronization for gamma band activity is incompatible with conventional views that higher frequency gamma band oscillations are likely generated by short-range neuronal circuits within the cortex, most likely involving inhibitory interneurons (Kopell 
et al., 2000; Whittington and Traub, 2003). The role of local inhibitory feedback in cortical gamma generation is partly supported by the observation that both VI and MI gamma frequency has been shown to be correlated with in-vivo measures of the inhibitory neurotransmitter GABA (Muthukumaraswamy et al., 2009; Gaetz et al., 2011) although a more recent MEG study found that only beta, and not gamma oscillations in MI were modulated by a $\mathrm{GABA}_{\mathrm{A}}$ receptor agonist (Muthukumaraswamy et al., 2012). In addition, recent evidence suggests that GABAergic mechanisms may also reflect long-range connectivity within the brain (Caputi et al., 2013). Litvak et al. (2012) applied coherence and Granger causality methods to movement-related gamma oscillations measured simultaneously in MI and STN in Parkinson patients to estimate long-range coupling and its directionality between these structures. They found evidence that the STN is driven by MI in the beta band and a weak effect for gamma activity showing the opposite directionality (STN phase-leading cortex). It is unclear what mechanisms might be involved in the latter, since STN activity would have to propagate via globus pallidus and thalamus back to MI, although there is recent evidence of direct projections from STN to MI (Degos et al., 2008). Thus, the role long-range cortical-striatal loops in the neurogenesis of cooccurring gamma bursts in MI and subcortical structures remains to be fully resolved.

The results of our reproducibility experiment, although requiring further replication, provide evidence that gamma band frequency is highly consistent within subjects (and hemispheres) over time, suggesting that an individual's motor cortex gamma is tuned to a preferred frequency, at least for the same movement. Interestingly, a recent MEG study demonstrated that the frequency of visual cortex gamma was positively correlated with the surface area of visual cortex stimulated, suggesting that interindividual variations in cortical anatomy determine gamma oscillation frequency. A similar mechanism might underlie motor cortex gamma given its dependence on the type of movement performed. For example, upper limb movements should activate a larger region of MI than lower limb movements, the latter showing significantly lower gamma frequency (Cheyne et al., 2008). However, this seems a less plausible explanation for the marked left-right symmetry of motor cortex gamma frequency, which, somewhat speculatively, may be more likely attributed to symmetrical conduction times or distances between cortical and subcortical structures, if not due to synchronization by common subcortical pathways. Our results do not lend support to previous observations of a decrease in motor gamma frequency with age (Muthukumaraswamy et al., 2009; Gaetz et al., 2011) as we observed no consistent changes in peak gamma frequency over a several year period in either subject. More detailed longitudinal studies may be required to establish the role of individual differences such as age in the tuning of motor cortex gamma frequencies.

These results also suggest that the facilitative effect of gamma band stimulation on movement might be enhanced by the use of individual's preferred gamma frequency. Tsang and colleagues (Tsang et al., 2012b) found that individualized gamma stimulation frequencies improved symptoms in Parkinson patients equally well as standard HF stimulation, although they did not directly compare non-preferred gamma frequencies. Similarly, transcranial alternating current stimulation of the motor cortex at exactly $70 \mathrm{~Hz}$ was shown to have a facilitative effect on movement (Joundi et al., 2012) and such effects might be enhanced by stimulation at the individual's preferred gamma frequency, although such effects will likely be effector specific.

\section{CONCLUSIONS}

What is the functional meaning of high-frequency gamma oscillations in normal motor control? The presence of gamma oscillations during virtually all types of movement supports the general finding they have a functional, or even facilitative role in motor output. We initially speculated that gamma activity at movement onset reflected a "disinhibition" of cortico-basal ganglia motor circuits via projections from MI to STN necessary to initiate movement (Cheyne et al., 2008). However, evidence that gamma activity in MI phase-lags coherent activity in subcortical structures (Lalo et al., 2008; Litvak et al., 2012) has led to speculations that motor cortex gamma might be the result of propagation of activity through the thalamus, which is also driven by brainstem nuclei related to arousal (Brucke et al., 2012). This, combined with the observation that subcortical movement-evoked gamma scaled with movement amplitude, led these authors to propose that motor cortex gamma synchronization reflects a non-specific momentary state of arousal during (or enabling) the initiation of movement, reflecting response "vigour" (Jenkinson et al., 2013). However, a recent study showed that machine learning algorithms were able to distinguish different patterns of activity recorded from ECoG in patients sufficiently to control a prosthetic arm, providing evidence that signatures for different movements may exist in the motor gamma response (Yanagisawa et al., 2012).

An alternative hypothesis, not entirely incompatible with the above observations, is that motor gamma oscillations may reflect efference copy mechanisms during movement that underlie our attention to movement execution. A recent theory of basal ganglia function proposed that two distinct cortico-striatal loops provide a mechanism for the computation of error signals between planned and executed movements (Morita et al., 2012). In this model, preparatory signals are transferred from direct pathway circuits to one involving cortical pyramidal cells that "drive the indirect pathway with signals representing the action currently being executed" (Shepherd, 2013, p. 284). In this context, cortical gamma oscillations may represent signals relayed upstream from the basal ganglia regarding planned movements, and constitute part of a network involved in the comparison of motor intentions with sensory feedback, allowing for discrimination between motor "plans" and actual executed movements. Thus, motor cortex gamma may not be imperative for actual motor control, but may provide a temporal encoding of planned movements, contributing to our conscious awareness of having performed an intended movement. This would also be compatible with the observation that motor gamma seems to encode specific features of executed movements, yet is variable in amplitude and timing for individual movements, and the degree to which it is correlated with movement performance. This, in turn, may 
reflect fluctuations in our awareness of our actions on a momentto-moment basis. Further studies of the relationship of cortical gamma oscillations to motor attention and performance may help further unravel the neurogenesis of cortical gamma oscillations and their role in motor control.

\section{REFERENCES}

Adjamian, P., Hadjipapas, A., Barnes, G. R., Hillebrand, A., and Holliday, I. E. (2008). Induced Gamma activity in primary visual cortex is related to luminance and not color contrast: an MEG study. J. Vis. 8, 1-7. doi: 10.1167/ 8.7 .4

Alegre, M., Alonso-Frech, F., Rodriguez-Oroz, M. C., Guridi, J., Zamarbide, I., Valencia, M., et al. (2005). Movement-related changes in oscillatory activity in the human subthalamic nucleus: ipsilateral vs. contralateral movements. Eur. J. Neurosci. 22, 2315-2324. doi: 10.1111/j.1460-9568.2005.04409.x

Amirnovin, R., Williams, Z. M., Cosgrove, G. R., and Eskandar, E. N. (2004). Visually guided movements suppress subthalamic oscillations in Parkinson's disease patients. J. Neurosci. 24, 11302-11306. doi: 10.1523/JNEUROSCI.3242-04.2004

Androulidakis, A. G., Kuhn, A. A., Chen, C. C., Blomstedt, P., Kempf, F., Kupsch, A., et al. (2007). Dopaminergic therapy promotes lateralized motor activity in the subthalamic area in Parkinson's disease. Brain 130, 457-468. doi: 10.1093/brain/awl358

Ball, T., Demandt, E., Mutschler, I., Neitzel, E., Mehring, C., Vogt, K., et al. (2008). Movement related activity in the high gamma range of the human EEG. Neuroimage 41, 302-310. doi: 10.1016/j.neuroimage.2008.02.032

Bauer, M., Oostenveld, R., Peeters, M., and Fries, P. (2006). Tactile spatial attention enhances gammaband activity in somatosensory cortex and reduces low-frequency activity in parieto-occipital areas. J. Neurosci. 26, 490-501. doi: 10.1523/JNEUROSCI.5228-04.2006

Bells, S., Gaetz, W., Bostan, A. C., and Cheyne, D. (2007). "Highfrequency gamma oscillations in primary motor cortex during voluntary movements," in Proceedings of the 13th Annual Conference on Human Brain Mapping (Chicago, IL).

Brovelli, A., Lachaux, J. P., Kahane, P., and Boussaoud, D. (2005). High gamma frequency oscillatory activity dissociates attention from intention in the human premotor cortex. Neuroimage 28, 154-164. doi: 10.1016/j.neuroimage.2005.05.045

Brown, P. (2003). Oscillatory nature of human basal ganglia activity: relationship to the pathophysiology of Parkinson's disease. Mov. Disord. 18, 357-363. doi: 10.1002/ mds. 10358

Brucke, C., Bock, A., Huebl, J., Krauss, J. K., Schonecker, T., Schneider, G. H., et al. (2013). Thalamic gamma oscillations correlate with reaction time in a $\mathrm{Go} /$ noGo task in patients with essential tremor. Neuroimage 75C, 36-45. doi: 10.1016/j.neuroimage.2013.02.038

Brucke, C., Huebl, J., Schonecker, T., Neumann, W. J., Yarrow, K., Kupsch, A., et al. (2012). Scaling of movement is related to pallidal gamma oscillations in patients with dystonia. J. Neurosci. 32, 1008-1019. doi: 10.1523/JNEUROSCI.3860-11.2012

Caputi, A., Melzer, S., Michael, M., and Monyer, H. (2013). The long and short of GABAergic neurons. Curr. Opin. Neurobiol. 23, 179-186. doi: 10.1016/j.conb.2013.01.021

Cheyne, D., Bakhtazad, L., and Gaetz, W. (2006). Spatiotemporal mapping of cortical activity accompanying voluntary movements using an event-related beamforming approach. Hum. Brain Mapp. 27, 213-229. doi: 10.1002/ hbm. 20178

Cheyne, D., Bells, S., Ferrari, P., Gaetz, W., and Bostan, A. C. (2008). Self-paced movements induce high-frequency gamma oscillations in primary motor cortex. Neuroimage 42, 332-342. doi: 10.1016/j.neuroimage.2008.04.178

Cheyne, D. O. (2013). MEG studies of sensorimotor rhythms: a review. Exp. Neurol. 245, 27-39. doi: 10.1016/j.expneurol.2012.08.030

Crone, N. E., Miglioretti, D. L., Gordon, B., and Lesser, R. P. (1998). Functional mapping of human sensorimotor cortex with electrocorticographic spectral analysis. II. Event-related synchronization in the gamma band. Brain 121(Pt 12), 2301-2315. doi: 10.1093/brain/121.12.2301

Dalal, S. S., Guggisberg, A. G., Edwards, E., Sekihara, K., Findlay, A. M., Canolty, R. T., et al. (2008).

\section{ACKNOWLEDGMENTS}

This work was supported by a grant from the Natural Sciences and Engineering Research Council of Canada to the first author (NSERC RGPIN 184018). The authors wish to thank Sonya Bells and Cecilia Jobst for assistance with data recordings and analysis.

Five-dimensional neuroimaging: localization of the time-frequency dynamics of cortical activity. Neuroimage 40, 1686-1700. doi: 10.1016/j.neuroimage.2008.01.023

Darvas, F., Scherer, R., Ojemann, J. G., Rao, R. P., Miller, K. J., and Sorensen, L. B. (2010). High gamma mapping using EEG. Neuroimage 49, 930-938. doi: 10.1016/j.neuroimage.2009.08.041

Degos, B., Deniau, J. M., Le Cam, J., Mailly, P., and Maurice, N. (2008). Evidence for a direct subthalamocortical loop circuit in the rat. Eur. J. Neurosci. 27, 2599-2610. doi: 10.1111/j.1460-9568.2008.06229.x

Engel, A. K., and Singer, W. (2001). Temporal binding and the neural correlates of sensory awareness. Trends Cogn. Sci. 5, 16-25. doi: 10.1016/S1364-6613(00)01568-0

Fries, P. (2009). Neuronal gamma-band synchronization as a fundamental process in cortical computation. Annu. Rev. Neurosci. 32, 209-224. doi: 10.1146/annurev.neuro.051508. 135603

Gaetz, W., Edgar, J. C., Wang, D. J., and Roberts, T. P. (2011). Relating MEG measured motor cortical oscillations to resting gamma-aminobutyric acid (GABA) concentration. Neuroimage 55, 616-621. doi: 10.1016/j.neuroimage.2010.12.077

Gaetz, W., Liu, C., Zhu, H., Bloy, L., and Roberts, T. P. (2013). Evidence for a motor gamma-band network governing response interference. Neuroimage 74, 245-253. doi: 10.1016/j.neuroimage.2013.02.013

Gaetz, W., Macdonald, M., Cheyne, D., and Snead, O. C. (2010). Neuromagnetic imaging of movement-related cortical oscillations in children and adults: age predicts post-movement beta rebound. Neuroimage 51, 792-807. doi: 10.1016/j.neuroimage.2010. 01.077

Gross, J., Baillet, S., Barnes, G. R., Henson, R. N., Hillebrand, A., Jensen, O., et al. (2013). Good practice for conducting and reporting MEG research. Neuroimage 65, 349-363. doi: 10.1016/j.neuroimage.2012.10.001

Hinkley, L. B., Nagarajan, S. S., Dalal, S. S., Guggisberg, A. G., and Disbrow, E. A. (2011). Cortical temporal dynamics of visually guided behavior. Cereb. Cortex 21, 519-529. doi: 10.1093/cercor/bhq102

Huo, X., Wang, Y., Kotecha, R., Kirtman, E. G., Fujiwara, H., Hemasilpin, N., et al. (2011). High gamma oscillations of sensorimotor cortex during unilateral movement in the developing brain: a MEG study. Brain Topogr. 23, 375-384. doi: 10.1007/s10548-010-0151-0

Jenkinson, N., Kuhn, A. A., and Brown, P. (2013). gamma oscillations in the human basal ganglia. Exp. Neurol. 245, 72-76. doi: 10.1016/j.expneurol.2012.07.005

Joundi, R. A., Jenkinson, N., Brittain, J. S., Aziz, T. Z., and Brown, P. (2012). Driving oscillatory activity in the human cortex enhances motor performance. Curr. Biol. 22, 403-407. doi: 10.1016/j.cub.2012.01.024

Jurkiewicz, M. T., Gaetz, W. C., Bostan, A. C., and Cheyne, D. (2006). Post-movement beta rebound is generated in motor cortex: evidence from neuromagnetic recordings. Neuroimage 32, 1281-1289. doi: 10.1016/j.neuroimage.2006.06.005

Kennedy, J. S., Singh, K. D., and Muthukumaraswamy, S. D. (2011). An MEG investigation of the neural mechanisms subserving complex visuomotor coordination. Int. J. Psychophysiol. 79, 296-304. doi: 10.1016/j.ijpsycho.2010.11.003

Kopell, N., Ermentrout, G. B., Whittington, M. A., and Traub, R. D. (2000). Gamma rhythms and beta rhythms have different synchronization properties. Proc. Natl. Acad. Sci. U.S.A. 97, 1867-1872. doi: 10.1073/pnas.97.4.1867

Lalo, E., Thobois, S., Sharott, A., Polo, G., Mertens, P., Pogosyan, A., et al. (2008). Patterns of bidirectional communication between cortex and basal ganglia during movement in patients with Parkinson disease. J. Neurosci. 28, 3008-3016. doi: 10.1523/JNEUROSCI.5295-07.2008

Litvak, V., Eusebio, A., Jha, A., Oostenveld, R., Barnes, G., Foltynie, T., et al. (2012). Movement-related changes in local and long-range synchronization in Parkinson's disease revealed by simultaneous magnetoencephalography and intracranial recordings. $J$ Neurosci 32, 10541-10553. doi: 10.1523/JNEUROSCI.0767-12.2012 
Miller, K. J., Leuthardt, E. C., Schalk, G., Rao, R. P., Anderson, N. R., Moran, D. W., et al. (2007). Spectral changes in cortical surface potentials during motor movement. J. Neurosci. 27, 2424-2432. doi: 10.1523/JNEUROSCI.3886-06.2007

Morita, K., Morishima, M., Sakai, K., and Kawaguchi, Y. (2012). Reinforcement learning: computing the temporal difference of values via distinct corticostriatal pathways. Trends Neurosci. 35, 457-467. doi: 10.1016/j.tins.2012.04.009

Muthukumaraswamy, S. D. (2010). Functional properties of human primary motor cortex gamma oscillations. J. Neurophysiol. 104, 2873-2885. doi: 10.1152/jn. 00607.2010

Muthukumaraswamy, S. D. (2013). High-frequency brain activity and muscle artifacts in MEG/EEG: a review and recommendations. Front. Hum. Neurosci. 7:138. doi: 10.3389/fnhum.2013.00138

Muthukumaraswamy, S. D., Edden, R. A., Jones, D. K., Swettenham, J. B., and Singh, K. D. (2009). Resting GABA concentration predicts peak gamma frequency and fMRI amplitude in response to visual stimulation in humans. Proc. Natl. Acad. Sci. U.S.A. 106, 8356-8361. doi: 10.1073/pnas.0900728106

Muthukumaraswamy, S. D., Myers, J. F., Wilson, S. J., Nutt, D. J., Lingford-Hughes, A., Singh, K. D., et al. (2012). The effects of elevated endogenous GABA levels on movement-related network oscillations. Neuroimage 66C, 36-41. doi: 10.1016/j.neuroimage. 2012.10 .054

Muthukumaraswamy, S. D., and Singh, K. D. (2013). Visual gamma oscillations: the effects of stimulus type, visual field coverage and stimulus motion on MEG and EEG recordings. Neuroimage 69, 223-230. doi: 10.1016/j.neuroimage.2012.12.038

Palva, S., Palva, J. M., Shtyrov, Y., Kujala, T., Ilmoniemi, R. J., Kaila,
K., et al. (2002). Distinct gammaband evoked responses to speech and non-speech sounds in humans. J. Neurosci. 22, RC211.

Pantev, C., Makeig, S., Hoke, M., Galambos, R., Hampson, S., and Gallen, C. (1991). Human auditory evoked gamma-band magnetic fields. Proc. Natl. Acad. Sci. U.S.A. 88, 8996-9000. doi: 10.1073/pnas.88.20.8996

Pfurtscheller, G., Graimann, B. Huggins, J. E., Levine, S. P., and Schuh, L. A. (2003). Spatiotemporal patterns of beta desynchronization and gamma synchronization in corticographic data during self-paced movement. Clin. Neurophysiol. 114, 1226-1236. doi: 10.1016/S1388-2457(03)00067-1

Robinson, S. E., and Vrba, J. (1999). Functional Neuroimaging by Synthetic Aperture Magnetometry. Sendai: Tohoku University Press.

Ross, B., Herdman, A. T., and Pantev, C. (2005). Stimulus induced desynchronization of human auditory $40-\mathrm{Hz}$ steady-state responses. J. Neurophysiol. 94, 4082-4093. doi: 10.1152/jn.00469.2005

Shepherd, G. M. (2013). Corticostriatal connectivity and its role in disease. Nat. Rev. Neurosci. 14, 278-291. doi: 10.1038/nrn3469

Swettenham, J. Muthukumaraswamy, S. D., and Singh, K. D. (2009). Spectral properties of induced and evoked gamma oscillations in human early visual cortex to moving and stationary stimuli. J. Neurophysiol. 102, 1241-1253. doi: 10.1152/jn.91044.2008

Szurhaj, W., Labyt, E., Bourriez, J. L., Kahane, P., Chauvel, P., Mauguiere, F., et al. (2006). Relationship between intracerebral gamma oscillations and slow potentials in the human sensorimotor cortex. Eur. J. Neurosci. 24, 947-954. doi: 10.1111/ j.1460-9568.2006.04876.x

Tallon-Baudry, C., and Bertrand, O. (1999). Oscillatory gamma activity in humans and its role in object representation. Trends Cogn. Sci. 3, 151-162. doi: 10.1016/S13646613(99)01299-1

Tecchio, F., Zappasodi, F., Porcaro, C., Barbati, G., Assenza, G., Salustri, C., et al. (2008). High-gamma band activity of primary hand cortical areas: a sensorimotor feedback efficiency index. Neuroimage 40, 256-264. doi: 10.1016/j. neuroimage.2007.11.038

Tsang, E. W., Hamani, C., Moro, E., Mazzella, F., Lozano, A. M., Hodaie, M., et al. (2012a). Movement related potentials and oscillatory activities in the human internal globus pallidus during voluntary movements. J. Neurol. Neurosurg. Psychiatry 83, 91-97. doi: 10.1136/jnnp.2011. 243857

Tsang, E. W., Hamani, C., Moro, E. Mazzella, F., Saha, U., Lozano, A. M., et al. (2012b). Subthalamic deep brain stimulation at individualized frequencies for Parkinson disease. Neurology 78, 1930-1938. doi: 10.1212/WNL.0b013e318259e183

Whitham, E. M., Pope, K. J., Fitzgibbon, S. P., Lewis, T., Clark, C. R., Loveless, S., et al. (2007). Scalp electrical recording during paralysis: quantitative evidence that EEG frequencies above $20 \mathrm{~Hz}$ are contaminated by EMG. Clin. Neurophysiol. 118 , 1877-1888. doi: 10.1016/j.clinph. 2007.04.027

Whittington, M. A., and Traub, R. D. (2003). Interneuron diversity series: inhibitory interneurons and network oscillations in vitro. Trends Neurosci. 26, 676-682. doi: 10.1016/j.tins.2003. 09.016

Wilson, T. W., Slason, E., Asherin, R., Kronberg, E., Reite, M. L., Teale, P. D., et al. (2010). An extended motor network generates beta and gamma oscillatory perturbations during development. Brain Cogn. 73, 75-84. doi: 10.1016/j. bandc.2010.03.001
Womelsdorf, T., and Fries, P. (2007). The role of neuronal synchronization in selective attention. Curr. Opin. Neurobiol. 17, 154-160. doi: 10.1016/j.conb.2007.02.002

Yanagisawa, T., Hirata, M., Saitoh, Y., Kishima, H., Matsushita, K., Goto, T., et al. (2012). Electrocorticographic control of a prosthetic arm in paralyzed patients. Ann. Neurol. 71, 353-361. doi: 10.1002/ana.22613

Yuval-Greenberg, S., Tomer, O., Keren, A. S., Nelken, I., and Deouell, L. Y. (2008). Transient induced gamma-band response in EEG as a manifestation of miniature saccades. Neuron 58, 429-441. doi: 10.1016/j.neuron.2008.03.027

Conflict of Interest Statement: The authors declare that the research was conducted in the absence of any commercial or financial relationships that could be construed as a potential conflict of interest.

Received: 19 April 2013; accepted: 27 August 2013; published online: 17 September 2013.

Citation: Cheyne D and Ferrari P (2013)

MEG studies of motor cortex gamma oscillations: evidence for a gamma "fingerprint" in the brain? Front. Hum. Neurosci. 7:575. doi: 10.3389/fnhum. 2013.00575

This article was submitted to the journal Frontiers in Human Neuroscience. Copyright (c) 2013 Cheyne and Ferrari. This is an open-access article distributed under the terms of the Creative Commons Attribution License (CC BY). The use, distribution or reproduction in other forums is permitted, provided the original author(s) or licensor are credited and that the original publication in this journal is cited, in accordance with accepted academic practice. No use, distribution or reproduction is permitted which does not comply with these terms. 